\title{
Rayleigh Wave in a Micropolar Elastic Medium with Impedance Boundary Conditions
}

\author{
Baljeet Singh \\ Department of Mathematics, Post Graduate Government College, Sector-11, Chandigarh - 160 011, India \\ Email: bsinghgc11@gmail.com
}

\begin{abstract}
In this paper, a problem on Rayleigh surface wave in an isotropic micropolar elastic solid half-space with impedance boundary conditions is investigated. It is assumed that the normal force traction, shear force traction and shear couple traction vary linearly with the normal displacement component, tangential displacement component and microrotation component multiplied by the frequency, where the impedance corresponds to the constants of proportionality. The linear governing equations of an isotropic micropolar elastic medium are solved for general surface wave solutions. The appropriate particular solutions satisfying the radiation conditions in a half-space of medium are applied at the free surface of the half-space with impedance boundary conditions. The secular equation for Rayleigh surface wave under impedance boundary conditions is derived in the explicit form. In the absence of impedance and microrotation, the secular equation reduces to classical secular equation for Rayleigh wave in an isotropic elastic half-space with traction free boundary conditions. The non-dimensional speed of propagation of Rayleigh wave is computed for an aluminium-epoxy composite as an example of a micropolar elastic solid and is shown graphically against nondimensional material constant, frequency and impedance parameters.
\end{abstract}

Keywords: Micropolar elasticity, Rayleigh wave, secular equation, wave speed.

\section{Introduction}

Eringen [1,2] developed the linear theory of micropolar elasticity. This theory has its possible applications in investigating deformation properties of solids, for which the purely elastic theory is inadequate. In particular, this theory is believed to be useful in investigation of materials consisting of bar-like molecules having microrotational effects and which support body and surface couples. Wood, fibres and corpuscles are some examples of such materials.

Surface waves are very useful in the fields of earthquake, geophysics and geodynamics. Due to slower attenuation of energy than that of body waves, the Rayleigh waves cause destruction to the structure. Rayleigh waves can travel along the surface of relatively thicker solid materials penetrating to a depth of one wave length. These waves are very sensitive to surface defects. Rayleigh waves are widely used for material characterization and to investigate the mechanical and structural properties of the objects. Lord Rayleigh [3] was the first to study the surface waves for an isotropic elastic solid. The study on surface waves in micropolar elasticity has been carried out by many researchers. Some of significant works are cited herein. For example, De and Sengupta $\lceil 4\rceil$ studied the surface waves in micropolar elastic media. Das and Sengupta $\lceil 5\rceil$ studied the surface waves in micropolar thermoelasticity under the influence of gravity. Nowinski [6] studied the surface waves in an elastic micropolar and microstretch medium with nonlocal cohesion. Kumar and Singh 「7〕 studied the Rayleigh wave in micropolar thermoelasticity with stretch. Sharma et al. [8] studied the propagation of Rayleigh surface waves in microstretch thermoelastic continua under inviscid fluid loadings. Kumar et al [9] studied the Rayleigh waves in isotropic microstretch thermoelastic diffusion solid half space. Recently Singh et al. [10] studied the Rayleigh wave in a transversely isotropic microstretch elastic solid half-space.

Impedance boundary conditions are a linear combination of the unknown functions and their derivatives prescribed on the boundary. Impedance boundary conditions are commonly used in various fields of physics like acoustics and electromagnetism. Rayleigh waves with impedance boundary conditions are significant in many fields of science and technology. However, few problems on Rayleigh 
wave with impedance boundary conditions are studied yet in literature. For example, Malischewsky [11] studied the Rayleigh waves with Tiersten's impedance boundary conditions and obtained a secular equation. Godoy et al. [12] studied the existence and uniqueness of Rayleigh waves with impedance boundary conditions. Vinh and Hue [13] investigated the propagation of Rayleigh waves in an orthotropic and monoclinic half-space with impedance boundary conditions. Recently, Singh [14] studied the Rayleigh wave in an isotropic generalized thermoelastic solid half-space with impedance boundary.

The present paper is motivated by the linear theory of micropolar elasticity formulated by Eringen [1]. The general surface wave solutions of the linear governing equations for an isotropic micropolar elastic body are obtained. The particular solutions which satisfy the radiation conditions in the half-space are obtained. These particular solutions satisfy the impedance boundary conditions and a secular equation of the Rayleigh surface wave in the half-space is obtained. The non-dimensional speed of the Rayleigh wave is computed and shown graphically against the frequency, non-dimensional parameter and impedance parameters for an aluminium-epoxy composite.

\section{Governing Equations and Solution}

A homogeneous, isotropic, micropolar elastic solid occupying the half-space is considered in an undisturbed state. The rectangular Cartesian coordinates are introduced, having the origin on the surface $z=0$ and the $z$-axis is chosen in the direction of increasing depth. A two-dimensional problem in $x-z$-plane is being discussed with the wave front parallel to the $y$-axis. Following Eringen [1], the constitutive and the field equations for an isotropic micropolar elastic medium in the absence of body forces and couples are

$$
\begin{gathered}
\sigma_{i j}=\lambda u_{r, r} \delta_{i j}+\mu\left(u_{i, j}+u_{j, i}\right)+\kappa\left(u_{j, i}-\varepsilon_{i j r} \phi_{r}\right), \\
m_{i j}=\alpha \phi_{r, r} \delta_{i j}+\beta \phi_{i, j}+\gamma \phi_{j, i}, \\
(\lambda+\mu) \nabla(\nabla \cdot \vec{u})+(\mu+\kappa) \nabla^{2} \vec{u}+\kappa \nabla \times \vec{\phi}=\rho \ddot{\vec{u}}, \\
(\alpha+\beta+\gamma) \nabla(\nabla \cdot \vec{\phi})-\gamma \nabla \times(\nabla \times \vec{\phi})+\kappa \nabla \times \vec{u}-2 \kappa \vec{\phi}=\rho j \ddot{\vec{\phi}},
\end{gathered}
$$

where $\lambda, \mu, \kappa, \alpha, \beta, \gamma$ are material constants, $\rho$ is the density, $j$ is the microinertia, $\vec{u}$ is the displacement vector, $\vec{\phi}$ is the microrotation vector, $\sigma_{i j}$ are the components of force stress and $m_{i j}$ are the components of couple stress. The superposed dots denote the time derivatives.

For the two-dimensional problem, let

$$
\vec{u}=\left(u_{1}, 0, u_{3}\right) \text { and } \vec{\phi}=\left(0, \phi_{2}, 0\right),
$$

where the displacement components $u_{1}$ and $u_{3}$ are written in terms of the potential functions $q(x, y, t)$ and $\psi(x, y, t)$ as

$$
u_{1}=\frac{\partial q}{\partial x}-\frac{\partial \psi}{\partial z}, u_{3}=\frac{\partial q}{\partial z}+\frac{\partial \psi}{\partial x},
$$

With the help of equations (5) and (6), the equations (3) and (4) reduce to

$$
\begin{gathered}
(\lambda+2 \mu+\kappa) \nabla^{2} q=\rho \ddot{q}, \\
(\mu+\kappa) \nabla^{2} \psi-\kappa \phi_{2}=\rho \ddot{\psi}, \\
\gamma \nabla^{2} \phi_{2}+\kappa \nabla^{2} \psi-2 \kappa \phi_{2}=\rho j \ddot{\phi}_{2},
\end{gathered}
$$

where the equations (8) and (9) are coupled in $\phi$ and $\psi$.

The general solution of equation (7) is obtained as

$$
q=\left(A_{1} e^{-m_{1} z}+A_{2} e^{m_{1} z}\right) e^{i k(x-c t)},
$$

where

$m_{1}^{2}=-k^{2}\left(\frac{\rho c^{2}}{\lambda+2 \mu+\kappa}-1\right)$. 
The general solutions of equations (8) and (9) are obtained as

$$
\begin{gathered}
\phi_{2}=\left(B_{1} e^{-m_{2} z}+B_{2} e^{-m_{3} z}+B_{3} e^{m_{2} z}+B_{4} e^{m_{3} z}\right) e^{i k(x-c t)}, \\
\psi=\left(\chi_{2} B_{1} e^{-m_{2} z}+\chi_{3} B_{2} e^{-m_{3} z}+\chi_{2} B_{3} e^{m_{2} z}+\chi_{3} B_{4} e^{m_{3} z}\right) e^{i k(x-c t)},
\end{gathered}
$$

where

$$
\begin{aligned}
& m_{2}^{2}+m_{3}^{2}=-k^{2}\left[\frac{\gamma P+N+\frac{\kappa R}{k^{2}}}{\gamma}\right], \quad m_{2}^{2} m_{3}^{2}=k^{4}\left[\frac{N P-\frac{\kappa R}{k^{2}}}{\gamma}\right], \\
& N=\rho j c^{2}-\gamma-\frac{2 \kappa}{k^{2}}, P=\frac{\rho c^{2}}{\mu+\kappa}-1, \quad R=\frac{\kappa}{\mu+\kappa} . \\
& \chi_{j}=-\frac{\left[\rho j c^{2}-2 \frac{\kappa}{k^{2}}+\gamma\left(\frac{m_{j}^{2}}{k^{2}}-1\right)\right]}{\kappa\left(\frac{m_{j}^{2}}{k^{2}}-1\right)},(j=2,3) .
\end{aligned}
$$

The particular solutions in half-space $(z>0)$, which satisfy the radiation conditions $q \rightarrow 0, \phi_{2} \rightarrow 0$, $\psi \rightarrow 0$ as $z \rightarrow-\infty$ are

$$
\begin{gathered}
q=A_{1} e^{-m_{1} z} e^{i k(x-c t)}, \\
\phi_{2}=\left(B_{1} e^{-m_{3} z}+B_{2} e^{-m_{4} z}\right) e^{i k(x-c t)}, \\
\psi=\left(\chi_{2} B_{1} e^{-m_{2} z}+\chi_{3} B_{2} e^{-m_{3} z}\right) e^{i k(x-c t)} .
\end{gathered}
$$

\section{$3 \quad$ Boundary Conditions}

Following Tiersten [15] and Malischewsky [12], the impedance boundary conditions at surface $z=0$ are

$$
\sigma_{z z}+\omega Z_{3} u_{3}=0, \quad \sigma_{z x}+\omega Z_{1} u_{1}=0, \quad m_{y z}+\omega Z_{2} \phi_{2}=0,
$$

where $\omega=k, c$ is circular frequency of wave, $k$ is wave number, $c$ is phase velocity; $Z_{1}, Z_{2}, Z_{3}$ are impedance real valued parameters, and

$$
\begin{aligned}
& \sigma_{z z}=\lambda u_{1,1}+(\lambda+2 \mu+\kappa) u_{3,3}, \\
& \sigma_{z x}=\mu u_{3,1}+(\mu+\kappa) u_{1,3}-\kappa \phi_{2}, \\
& m_{y z}=\beta \frac{\partial \phi_{2}}{\partial z} .
\end{aligned}
$$

The particular solutions (13) to (15) satisfy the impedance boundary conditions (16) at $\mathrm{z}=0$ and we obtain the following desired secular equation of Rayleigh wave

$$
\begin{aligned}
& \left(\frac{\beta}{\mu} \frac{m_{3}}{k}-X Z_{2}^{*}\right)\left(\frac{\lambda}{\mu}-\frac{\lambda+2 \mu+\kappa}{\mu} \frac{m_{1}^{2}}{k^{2}}+X Z_{3}^{*} \frac{m_{1}}{k}\right)\left[\chi_{2}\left(1+\frac{\mu+\kappa}{\mu} \frac{m_{2}^{2}}{k^{2}}-X Z_{1}^{*} \frac{m_{2}}{k}\right)+\frac{\kappa}{k^{2}}\right]- \\
& \left(\frac{\beta}{\mu} \frac{m_{2}}{k}-X Z_{2}^{*}\right)\left(\frac{\lambda}{\mu}-\frac{\lambda+2 \mu+\kappa}{\mu} \frac{m_{1}^{2}}{k^{2 ?}}+X Z_{3}^{*} \frac{m_{1}}{k}\right)\left[\chi_{3}\left(1+\frac{\mu+\kappa}{\mu} \frac{m_{3}^{2}}{k}-X Z_{1}^{*} \frac{m_{3}}{k}\right)+\frac{\kappa}{k}\right]+ \\
& \chi_{2}\left(\frac{\beta}{\mu} \frac{m_{3}}{k}-X Z_{2}^{*}\right)\left(\frac{2 \mu+\kappa}{\mu} \frac{m_{2}}{k}-X Z_{3}^{*}\right)\left(\frac{2 \mu+\kappa}{\mu} \frac{m_{1}}{k}-X Z_{1}^{*}\right)- \\
& \chi_{3}\left(\frac{\beta}{\mu} \frac{m_{2}}{k}-X Z_{2}^{*}\right)\left(\frac{2 \mu+\kappa}{\mu} \frac{m_{3}}{k}-X Z_{3}^{*}\right)\left(\frac{2 \mu+\kappa}{\mu} \frac{m_{1}}{k}-X Z_{1}^{*}\right)=0,
\end{aligned}
$$

where 


$$
X=\sqrt{\frac{\rho c^{2}}{\mu}}, Z_{1}^{*}=\frac{Z_{1}}{\sqrt{\rho \mu}}, Z_{2}^{*}=\frac{Z_{2}}{\sqrt{\rho \mu}}, Z_{3}^{*}=\frac{Z_{3}}{\sqrt{\rho \mu}} .
$$

\section{Particular Cases}

(i). In absence of impedance parameters, the secular equation (17) reduces to

$$
\begin{aligned}
& \left(\frac{\lambda}{\mu}-\frac{\lambda+2 \mu+\kappa}{\mu} \frac{m_{1}^{2}}{k^{2 ?}}\right)\left[\frac{m_{3}}{k}\left(\chi_{2}+\chi_{2} \frac{\lambda+\kappa}{\mu} \frac{m_{2}^{2}}{k}+\frac{\kappa}{k}\right)-\frac{m_{2}}{k}\left(\chi_{3}+\chi_{3} \frac{\lambda+\kappa}{\mu} \frac{m_{3}^{2}}{k}+\frac{\kappa}{k}\right)\right]+ \\
& \left(\chi_{2}-\chi_{3}\right) \frac{m_{1}}{k} \frac{m_{2}}{k} \frac{m_{3}}{k}\left(\frac{2 \mu+\kappa}{\mu}\right)^{2}=0
\end{aligned}
$$

which is the secular equation for a Rayleigh wave in an isotropic micropolar elastic solid half-space with traction free boundary.

(ii). In absence of microrotation, the secular equation (17) reduces to

$$
\left(\frac{\lambda}{\mu}-\frac{\lambda+2 \mu}{\mu} \frac{m_{1}^{2}}{k^{2 ?}}+X Z_{3}^{*} \frac{m_{1}}{k}\right)\left(-1-\frac{m_{2}^{2}}{k}+X Z_{1}^{*} \frac{m_{2}}{k}\right)-\left(2 \frac{m_{1}}{k}-X Z_{1}^{*}\right)\left(2 \frac{m_{2}}{k}-X Z_{3}^{*}\right)=0,
$$

which is the secular equation of Rayleigh wave in an isotropic elastic solid half-space with impedance boundary conditions. If we put $Z_{3}^{*}=0$ in equation (19), then the resulting secular equation fairly agrees with those obtained by Godoy et al. [12]

(iii). In absence of impedance parameters and microrotation, the frequency equation (17) reduces to

$$
\left(2-\frac{\rho c^{2}}{\mu}\right)^{2}=4 \sqrt{\left(1-\frac{\rho c^{2}}{+2 \mu}\right)} \sqrt{\left(1-\frac{\rho c^{2}}{\mu}\right)}
$$

which is the same as obtained by Lord Rayleigh [3].

\section{Numerical Results and Discussion}

Following Gauthier [16], the physical constants of aluminium-epoxy composite as an example of an isotropic micropolar solid are

$$
\begin{aligned}
& \lambda=7.59 \times 10^{11} \text { dyne } / \mathrm{cm}^{2}, \mu=1.89 \times 10^{11} \text { dyne } / \mathrm{cm}^{2}, \kappa=0.0149 \times 10^{11} \text { dyne } / \mathrm{cm}^{2}, \\
& \gamma=0.268 \times 10^{11} \text { dyne }, j=0.0196 \mathrm{~cm}^{2}, \rho=2.19 \mathrm{gm} / \mathrm{cm}^{3}, j k^{2}=0.1 .
\end{aligned}
$$

For above physical constants, the non-dimensional speed $\sqrt{\rho c^{2} / \mu}$ of the Rayleigh wave is computed from equation (17) for a particular range of non-dimensional material constant $(\kappa / \mu)$, the frequency $(\omega)$ and impedance parameters $Z_{1}^{*}, Z_{2}^{*}$ and $Z_{3}^{*}$.

The non-dimensional speed of the Rayleigh wave is plotted in Figure 1 against non-dimensional constant $(\kappa / \mu)$ varying from 0 to 0.1 , when $\omega=10 \mathrm{~Hz}$. The non-dimensional speed increases linearly with the increase in value of $\kappa / \mu$. The comparison of the solid line with other solid lines with center symbols in Figure 1 shows the effect of impedance and material constants on non-dimesional wave speed. The non-dimensional speed of the Rayleigh wave is plotted in Figure 2 against the frequency $(\omega)$ varying from $2 \mathrm{~Hz}$ to $20 \mathrm{~Hz}$. For lower range of frequency, the non-dimensional speed increases sharply, whereas it increases slowly for higher range of frequency. The comparison of the solid line with other solid lines with center symbols in Figure 2 shows the effect of impedance and frequency on nondimesional wave speed. 


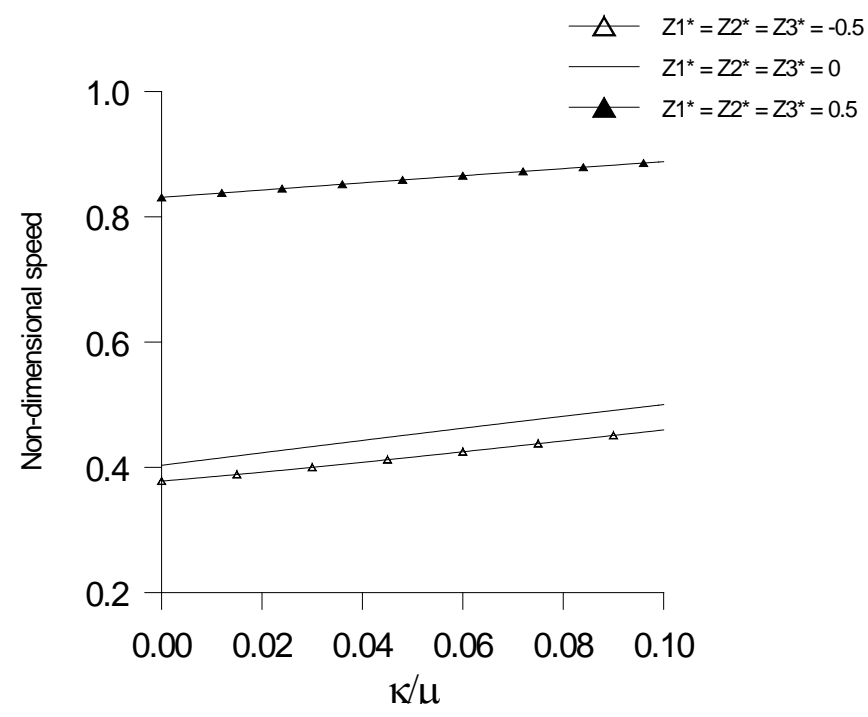

Figure 1. Variations of the non-dimensional speed $\sqrt{\rho c^{2} / \mu}$ of the Rayleigh wave against the non-dimensional constant $(\kappa / \mu)$, when $\omega=10 \mathrm{~Hz}$.

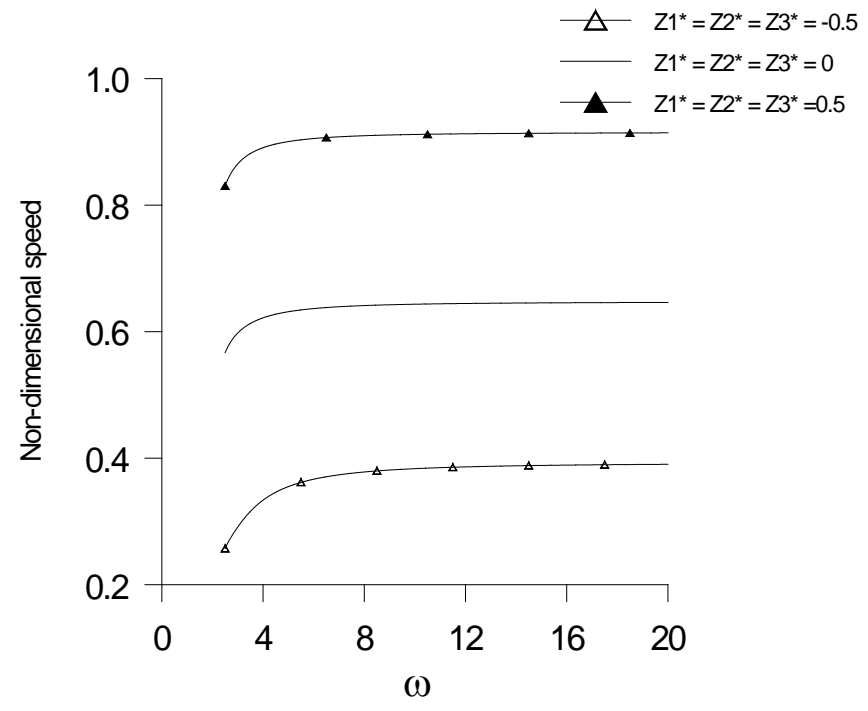

Figure 2. Variation of the non-dimensional speed $\sqrt{\rho c^{2} / \mu}$ of the Rayleigh wave against the frequency $(\omega)$

The variation of the non-dimensional speed of the Rayleigh wave against the impedance parameter $Z_{1}^{*}$ is shown graphically in Figure 3 when $Z_{2}^{*}=Z_{3}^{*}=0.5$. For $\omega=5,10$ and 15 , it increases gradually as $\left(Z_{1}^{*}\right)$ varies from -2 to 1 . The comparison of solid, small dashed and long dashed curves in Figure 3 shows the effect of frequency and impedance on non-dimensional wave speed of Rayleigh wave. The variation of the non-dimensional speed of the Rayleigh wave against the impedance parameter $\left(Z_{2}^{*}\right)$ is shown graphically in Figure 4, when $\omega=10 \mathrm{~Hz}$. For range $-20 \leq Z_{2}^{*}<0$, it decreases very slowly. There is a sudden fall in value of wave speed at $Z_{2}^{*}=0$. It increases very slowly for range $0<Z_{2}^{*} \leq 20$. The comparison of different curves in Figure 4 shows the effect of different impedance parameters on wave speed. The variation of the non-dimensional speed of the Rayleigh wave against the impedance parameter $\left(Z_{3}^{*}\right)$ is shown graphically in Figure 5 , when $\omega=10 \mathrm{~Hz}$. For the range $-2 \leq Z_{3}^{*} \leq 1$, it first decreases to its minimum value and then increases. The comparison of different curves in Figure 5 shows the effect of different impedance parameters on wave speed. 


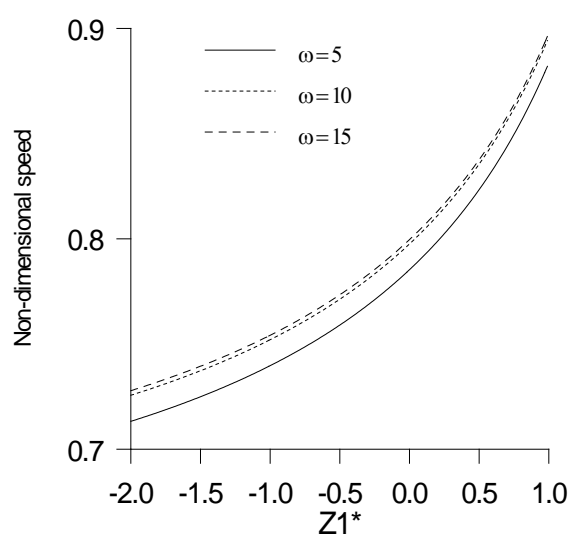

Figure 3. Variation of the non-dimensional speed $\sqrt{\rho c^{2} / \mu}$ of the Rayleigh wave against the impedance parameter $\left(Z_{1}^{*}\right)$ when $Z_{2}^{*}=Z_{3}^{*}=0.5$

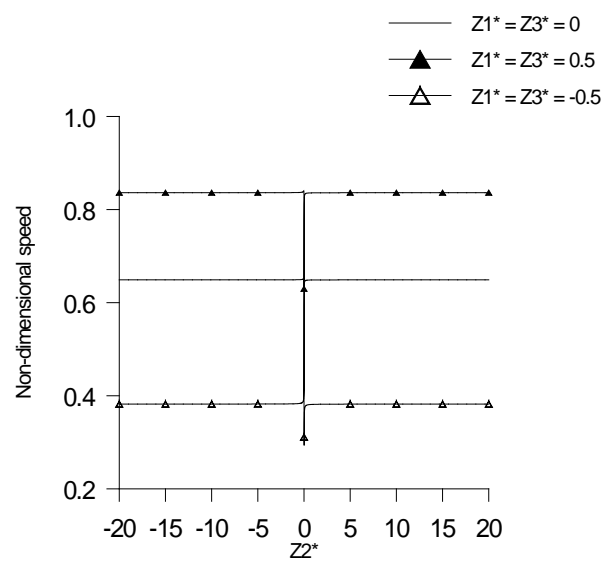

Figure 4. Variation of the non-dimensional speed $\sqrt{\rho c^{2} / \mu}$ of the Rayleigh wave against the impedance parameter $\left(Z_{2}^{*}\right)$, when $\omega=10 \mathrm{~Hz}$.

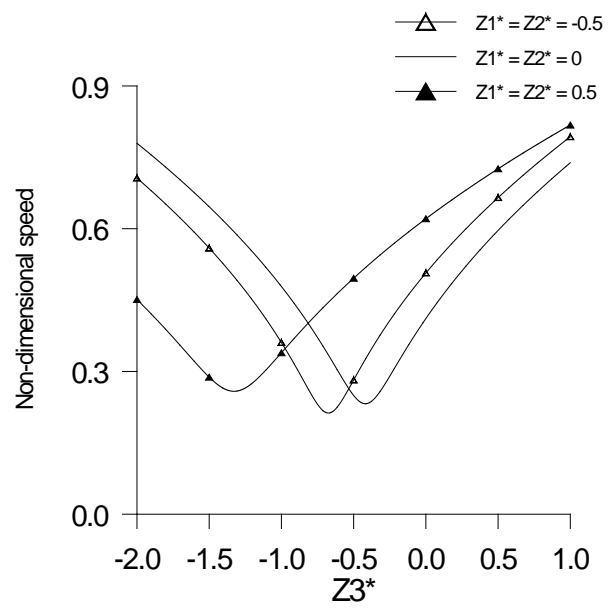

Figure 5. Variation of the non-dimensional speed $\sqrt{\rho c^{2} / \mu}$ of the Rayleigh wave against the impedance parameter $\left(Z_{3}^{*}\right)$, when $\omega=10 \mathrm{~Hz}$. 


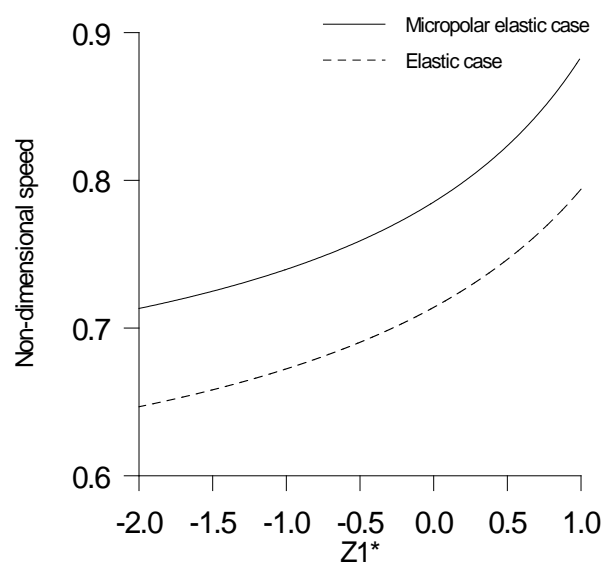

Figure 6. Effect of microrotation and impedance parameter $Z_{1}^{*}$ on variation of the non-dimensional speed $\sqrt{\rho c^{2} / \mu}$ of the Rayleigh wave.

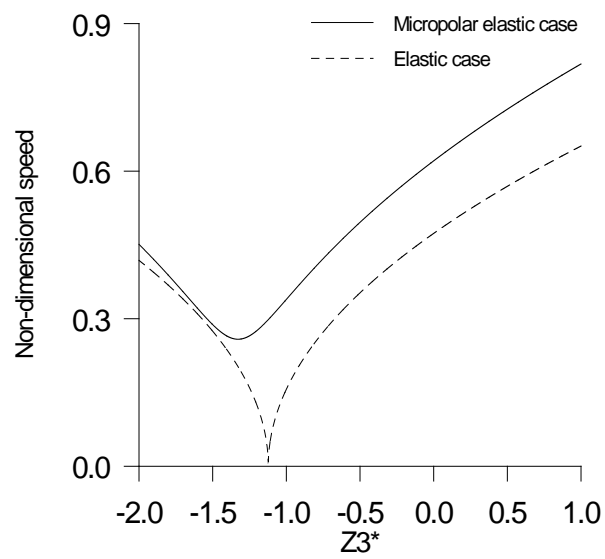

Figure 7. Effect of microrotation and impedance parameter $Z_{3}^{*}$ on variation of the non-dimensional speed $\sqrt{\rho c^{2} / \mu}$ of the Rayleigh wave.

From above discussion, it is observed that

(i). For a fixed value of frequency, the non-dimensional wave speed of Rayleigh wave increases linearly with the increase in value of non-dimensional material constant.

(ii). The non-dimensional wave speed increases with the increase in frequency. However this increase is significant in lower range of frequency.

(iii). The non-dimensional wave speed increases due to the presence of microrotation in an elastic solid.

(iv). The non-dimensional wave speed increases gradually in given range of impedance parameter $Z_{1}^{*}$, whereas it oscillates in given ranges of impedance parameters $Z_{2}^{*}$ and $Z_{3}^{*}$.

\section{Conclusion}

The governing equations of the linear, isotropic and homogeneous micropolar elastic medium are solved for general surface wave solutions. Particular solutions which satisfy the radiation conditions in halfspace are obtained. A secular equation of Rayleigh surface wave in a micropolar elastic solid half-space with impedance boundary conditions is derived. In absence of impedance and microrotation, the secular equation reduces to classical equation as obtained by Lord Rayleigh [3] for an isotropic elastic solid halfspace with traction free boundary conditions. The theoretical results are illustrated with the help of material aluminium-epoxy composite as an example of micropolar elastic solid. The non-dimensional speed of the Rayleigh wave is computed for a given range of the frequency, non-dimensional constant 
$(\kappa / \mu)$ and impedance parameters. From theory and numerical analysis, it is observed that the nondimensional speed of the Rayleigh wave depends upon material parameters, frequency, microrotation and impedance parameters.

\section{References}

1. A. C. Eringen, "Linear theory of micropolar elasticity", Journal of Mathematics and Mechanics, vol. 15, pp. 909-924, 1966.

2. A. C. Eringen, "Theory of micropolar elasticity". Fracture, vol. 2, Academic Press, New York, 1968.

3. L. Rayleigh, "On waves propagated along the plane surface of an elastic solid", Proceedings of Royal Society of London, Series A, vol. 17, pp. 4-11, 1885.

4. S. N. De and P. R. Sengupta, "Surface waves in micropolar elastic media", Bull. Acad. Pol. Sci. Ser. Sci. Technol., vol. 22, pp. 137-146, 1974.

5. P. S. Das and P. R. Sengupta, "Surface waves in micropolar thermoelastcity under the influence of gravity", Proceeding of Indian National Science Academy, vol. 56, pp. 459-471, 1990.

6. J. L. Nowinski, "On the surface waves in an elastic micropolar and microstretch medium with nonlocal cohesion", Acta Mechanica, vol. 96, pp. 97-108, 1993.

7. R. Kumar and B. Singh, "Wave propagation in micropolar generalized thermoelastic body with stretch", Proceeding of Indian Academy of Sciences (Mathematical Sciences), vol.106, pp. 183-199, 1996.

8. J. N. Sharma, S. Kumar and Y. D. Sharma, "Propagation of Rayleigh surface waves in microstretch thermoelastic continua under inviscid fluid loadings", Journal of Thermal Stresses, vol. 31, pp. 18-39, 2008.

9. R. Kumar, S. Ahuja and S. K. Garg, "Rayleigh waves in isotropic microstretch thermoelastic diffusion solid half space", Latin American Journal of Solids and Structures, vol. 11, pp. 299-319, 2014.

10. B. Singh, R. Sindhu and J. Singh, "Rayleigh surface waves in a transversely isotropic microstretch elastic solid half-space", Journal of . Multidisciplinary Engineering Science and Technology, vol. 2, pp. 1742-1746, 2015.

11. P. G. Malischewsky, "Surface waves and discontinuities", Elsevier, Amsterdam, 1987.

12. E. Godoy, M. Durn and J.-C. Ndlec, "On the existence of surface waves in an elastic half-space with impedance boundary conditions", Wave Motion, vol. 49, pp. 585-594, 2012.

13. P.C. Vinh and T.T.T. Hue, "Rayleigh waves with impedance boundary conditions in anisotropic solids", Wave Motion, vol. 51, pp. 1082-1092, 2014.

14. B. Singh, "Rayleigh wave in a thermoelastic solid half-space with impedance boundary conditions", Meccanica, vol. 51, pp. 1135, 2016.

15. H. F. Tiersten, "Elastic surface waves guided by thin films", Journal of Applied Physics, vol. 40, pp. 770-789, 1969.

16. R. D. Gauthier, "Experimental investigation on micropolar media", in Mechanics of Micropolar Media (eds. O. Brulin and R.K.T. Hsieh), World Scientific, Singapore, 1982. 